University of Wollongong

Research Online

Faculty of Engineering - Papers (Archive)

Faculty of Engineering and Information

Sciences

June 2001

\title{
Time-Dependent Particle Transport through Granular Filters
}

M. Locke

University of Wollongong

Buddhima Indraratna

University of Wollongong, indra@uow.edu.au

G. Adikari

Snowy Mountains Engineering Corporation, Australia

Follow this and additional works at: https://ro.uow.edu.au/engpapers

Part of the Engineering Commons

https://ro.uow.edu.au/engpapers/180

\section{Recommended Citation}

Locke, M.; Indraratna, Buddhima; and Adikari, G.: Time-Dependent Particle Transport through Granular Filters 2001.

https://ro.uow.edu.au/engpapers/180

Research Online is the open access institutional repository for the University of Wollongong. For further information contact the UOW Library: research-pubs@uow.edu.au 


\title{
Time-Dependent Particle Transport through Granular Filters
}

\author{
By Mark Locke, ${ }^{1}$ Buddhima Indraratna, ${ }^{2}$ Member, ASCE, and Gamini Adikari ${ }^{3}$
}

\begin{abstract}
This paper describes an analytical model of filtration for granular media, based on the mechanics of particle migration under hydraulic loads. A new equation to predict the probability of particle movement through a 3D network model of the filter voids has been developed. Void constriction sizes are determined based on the particle-size distribution and relative density of the filter. An important new development is the differentiation between particles that form part of the filter structure and fine particles that are loose within the filter voids, or coarse particles that are enmeshed in a matrix of fines. The rate of particle erosion and transport is governed by the consideration of mass and momentum conservation. The model describes the time-dependent change of flow rate and base and filter particle-size distribution, porosity, and permeability. The model has application in the design of granular filters for noncohesive uniform, well-, and broadly graded base and filter materials.
\end{abstract}

\section{INTRODUCTION}

Granular filters are used where it is necessary to protect a soil from erosion due to seepage of water. A correctly designed filter will retain any eroded soil particles while allowing seepage water to flow, thus preventing erosion of the protected soil. Empirical methods based on particle-size ratios are commonly used for the design of granular filters. These empirical guidelines are developed through extensive laboratory tests, whereby the stability of various base-soil-filter combinations is evaluated under different hydraulic gradients (Sherard et al. 1984; Delgado Ramos 2000). However, the mechanisms of filtration, interface behavior, and time-dependent changes that occur within the filter medium cannot be described by empirical criteria. In contrast, mathematical models can be formulated to explain the fundamental physics of particle erosion, transport, and retention, within a framework of well-defined geohydraulic constraints.

Silveira (1965) used probabilistic methods to examine migration of base-soil particles into filters, based on the observation that a particle can move from one pore to the next if the particle is smaller than the opening between these pores, called the pore constriction. A probabilistic comparison of the base-soil particle sizes and filter constriction size distribution lead to an estimate of the infiltration depth into clean filters. Humes (1996) adapted the Silveira (1965) model to more accurately describe broadly graded materials. Witt (1993) developed a 3D pore network model of spheres (pores) interconnected by pipes (pore constrictions). This model assumes that, for each pore, there are a number of possible exits through pore constrictions and the largest pore constriction from each pore determines whether a particle can move from the pore. Schuler (1996) adopted a similar 3D void network model and used a Monte Carlo simulation to examine the infiltration depth of base-soil particles into the filter model. These models do not incorporate the time-dependent changes in filter void constriction sizes as particles are captured. Indraratna and Vafai (1997) considered a number of elements at the base-filter

${ }^{1}$ Doctoral Candidate, Facu. of Engrg., Univ. of Wollongong, NSW, Australia, 2522.

${ }^{2}$ Prof. of Civ. Engrg., Facu. of Engrg., Univ. of Wollongong, NSW, Australia, 2522.

${ }^{3}$ Prin. Engr. Dams, Snowy Mountains Engineering Corp., P.O. Box 559 Carnegie VIC, Australia, 3163.

Note. Discussion open until November 1, 2001. To extend the closing date one month, a written request must be filed with the ASCE Manager of Journals. The manuscript for this paper was submitted for review and possible publication on May 31, 2000; revised January 5, 2001. This paper is part of the Journal of Geotechnical and Geoenvironmental Engineering, Vol. 127, No. 6, June, 2001. C)ASCE, ISSN 1090-0241/01/ 0006-0521-0529/\$8.00 + \$.50 per page. Paper No. 22388. interface, where the movement of particles is modeled by a finite-difference procedure based on conservation of mass and momentum. The analysis predicts the gradual change in particle-size distribution, permeability, and porosity of the materials with time, hence, predicting the time-dependent changes at the base-filter interface for the entire particle-size range during the life of the filter. A more detailed discussion of analytical models for granular filters can be found in Indraratna and Locke (2000). Reddi (1997) has reviewed other models developed to describe particle transport in soils and described their applications in practice.

Existing analytical models of filtration have certain limitations. Many adopt simplified pore models that are only applicable to a limited range of filter materials. Very few models consider the time rate of formation of a stable filter interface. Instead, the models only determine the amount of particle infiltration into a clean filter while ignoring the change in filter void sizes and permeability due to capture of base-soil particles. By considering the time-dependent changes, the gradual development of a self-filtration zone is described, where retained coarse particles are then able to retain finer particles. Self-filtration cannot be described without considering these time-dependent processes. These time-dependent predictions are very useful to the designer, predicting the rate of internal erosion will determine the potential warning time before failure of a structure or the expected time after construction before a successful filter prevents further particle transport. This paper describes a revised particle-transport model for filtration of noncohesive soils, which significantly extends the previously proposed analytical model by Indraratna and Vafai (1997) for particle transport. The entire particle-transport model includes a filter void model based on a 3D pore network model and principles of conservation of mass and momentum describing the movement of soil particles through the filter voids. The filter void model incorporates a probabilistic analysis of basesoil particle sizes and filter void sizes to determine the depth a particle can infiltrate into the filter. The base soil and filter are divided into a number of discrete elements in the direction of flow at the base-soil-filter interface, and the time-dependent changes occurring in these elements is modeled.

\section{FILTER VOID MODEL}

A granular soil is a $3 \mathrm{D}$ collection of particles that form pores of different sizes and shapes having constrictions between the pores, which may be of different sizes, shapes, and orientations. A model of these voids is required for the analysis. Schuler (1996) developed a regular cubic network model of pores and constrictions, shown in Fig. 1(a). Each pore void has six exits or constrictions [Fig. 1(b)]. A particle that has 


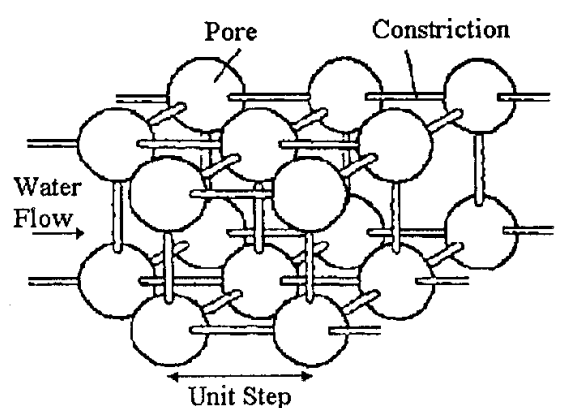

(a)

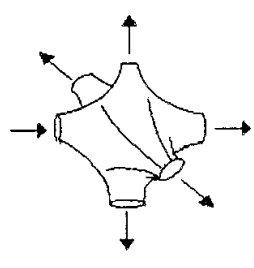

(b)
FIG. 1. (a) Cubic Network Pore Model [after Schuler (1996)]; (b) Single Pore with Six Constrictions

entered a pore through a constriction may exit the pore through any of the other five constrictions, provided the particle is smaller than the constriction diameter. This void model will be adopted in the current analysis.

\section{Constriction Size Distribution (CSD)}

In the filter void model, the pore constrictions form the smallest link between pores, capturing moving particles. Hence, the important geometric factor for modeling nonuniform granular filters is the pore CSD. An assumption made here is that the CSD is dependent on the particle-size distribution (PSD) of the filter determined by the surface area of the particles, as proposed by Humes (1996). Constriction sizes can be estimated by considering one of the two geometric conditions shown in Fig. 2. These two geometric conditions represent, in two dimensions, the most-dense and the leastdense packing condition of the filter particles. The diameter of a circle that can fit between the three or four filter particles represents the size of the constriction formed by these particles. A method to find the least-dense CSD [Fig. 2(b)] is described by Silveira et al. (1975). This method is also outlined by Indraratna and Locke (2000) and will not be repeated here.

Considering the most-dense particle packing, where the constriction is formed by three tangent spheres of diameters $D_{i}$, $D_{j}$, and $D_{k}$ [Fig. 2(a)], the theory of standard mean error can be used to estimate the constriction diameter $D_{\mathrm{v}}$, as given by

$$
\begin{aligned}
& \left(\frac{2}{D_{i}}\right)^{2}+\left(\frac{2}{D_{j}}\right)^{2}+\left(\frac{2}{D_{k}}\right)^{2}+\left(\frac{2}{D_{\mathrm{v}}}\right)^{2} \approx 0.5\left[\left(\frac{2}{D_{i}}\right)+\left(\frac{2}{D_{j}}\right)\right. \\
& \left.+\left(\frac{2}{D_{k}}\right)+\left(\frac{2}{D_{\mathrm{v}}}\right)\right]^{2}
\end{aligned}
$$

Humes (1996) considered the frequency of the three filter particle sizes (from the filter PSD by surface area), to determine the frequency of different constriction sizes, leading to the CSD. The frequency $P_{\mathrm{v}}$ of the constriction size $D_{\mathrm{v}}$ is a function of the frequency of the three particle diameters $\left(P_{i}, P_{j}\right.$, and $\left.P_{k}\right)$ taken from the PSD. The value of $P_{\mathrm{v}}$ is calculated with (2), where $r_{i}, r_{j}$, and $r_{k}$ represent the number of times the particle diameters $D_{i}, D_{j}$, and $D_{k}$ appear in the combination of three particles being considered. Hence $r_{i}, r_{j}$, and $r_{k}=0,1,2$, or 3 and $r_{i}+r_{j}+r_{k}=3$. The CSD that is most dense $\operatorname{CSD}_{M D}$ is then a cumulative distribution of constriction sizes $D_{\vee}$ and the corresponding frequency $P_{\mathrm{v}}$

$$
P_{\mathrm{v}}=\frac{3 !}{r_{i} ! r_{j} ! r_{k} !}\left(P_{i}\right)^{r i} \cdot\left(P_{j}\right)^{r j} \cdot\left(P_{k}\right)^{r k}
$$

The two geometric cases shown in Fig. 2 represent the extremes of relative density. Real filters are unlikely to exist either in the most-dense or least-dense states but rather at some intermediate state. Hence, a more representative model would

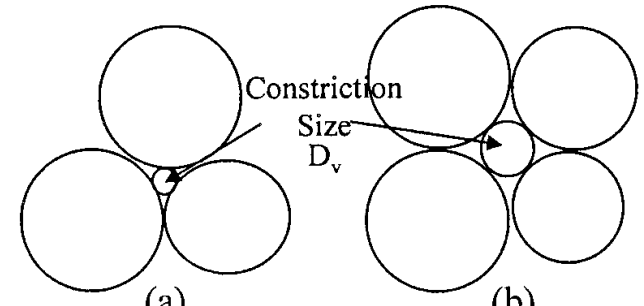

(a)

(b)

FIG. 2. Pore Constriction Size for (a) Most-Dense State; (b) LeastDense State [after Terzaghi (1922)]

consider the filter void sizes based on the filter PSD and relative density. Schuler (1996) has examined the CSD of a soil at varying relative density and proposed that all the CSD curves have the same shape. However, Giroud (1996) suggested that, in certain locations within a medium dense to dense granular material, a number of particles will group together to form a maximum density arrangement. The assumption is made that the coarser pore constrictions increase in size proportionally with a decrease in relative density $R_{d}$ between the most-dense and least-dense packing models and the smallest constrictions are equal in size to the most-dense packing arrangement, as shown in (3). The CSD is divided into $n$ discrete portions. The integer $i$ represents these discrete portions of the CSD such that $i / n$ is the fraction of constrictions finer than constriction diameter $D_{\mathrm{v}, i}$

$$
D_{\vee, i}=D_{V M D, i}+\frac{i}{n}\left(1-R_{d}\right)\left(D_{V L D, i}-D_{V M D, i}\right), \quad i=1,2, \ldots, n
$$

where $D_{V M D, i}$ and $D_{V L D, i}=i / n \%$ coarsest constrictions from the most-dense and least-dense CSDs, respectively.

\section{Constriction Sizes of Broadly Graded Materials}

Broadly graded base and filter materials present a further problem in modeling, particularly for the determination of the CSD. In some cases, the fine particles may not contribute to the stable structure of the granular material but may be loose within the pores formed by the stable skeleton of larger particles. In the opposite case, the coarse particles may be enmeshed in a matrix of fines. The fines fill the voids between coarse particles and large constrictions are filled. In either case, the filter PSD should be truncated to discount particles that do not contribute to the CSD.

Skempton and Brogan (1994) defined an ideal grading curve, represented by (4), where $S_{n}$ is the mass fraction passing diameter $D_{n}$ and $D_{\max }$ is the largest particle diameter. The ideal curve is shown in Fig. 3. This curve describes the PSD of the

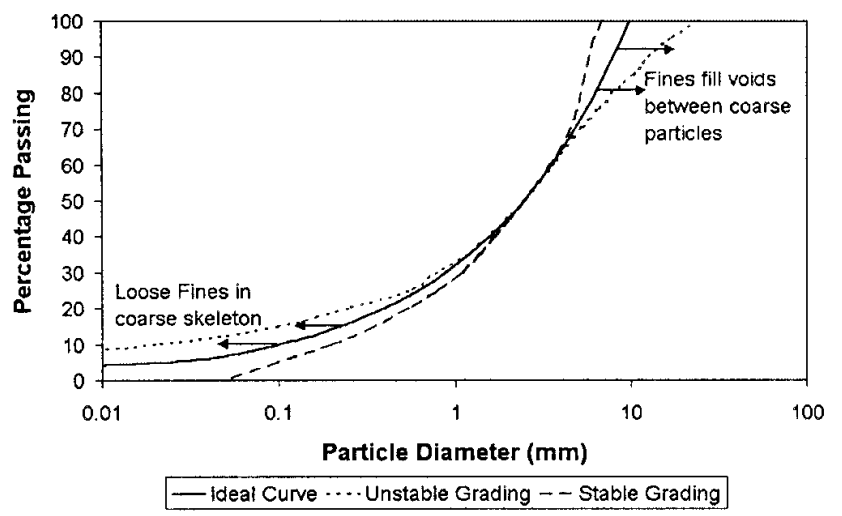

FIG. 3. Ideal Curve and Stable and Unstable Gradings for Broadly Graded Filters 
most-dense possible particle arrangement, where fine particles exactly fit between the voids formed by coarser particles. It is assumed that, if there are more fine particles than predicted by this ideal curve (i.e., the fine fraction of the "unstable" curve in Fig. 3), some fine particles will be loose within the soil skeleton and do not contribute to the CSD. If the coarse particles are larger than predicted by the ideal curve (i.e., the coarse fraction of the "unstable" curve in Fig. 3), the voids formed by these coarse particles will be filled by finer particles

$$
S_{n}=\sqrt{\frac{D_{n}}{D_{\max }}}
$$

Eq. (4) can be rewritten to relate different particle sizes within the ideal grading curve, eliminating $D_{\max }$

$$
D_{n}=D_{m}\left(\frac{S_{n}}{S_{m}}\right)^{2}
$$

where $S_{m}=$ fraction passing diameter $D_{m}$. As described above, fine particles are loose within the soil skeleton if $D_{n}$ is finer than predicted by (5). By substituting $m=2 n$, particles finer than $D_{n}$ are loose if

$$
D_{n}<\frac{D_{2 n}}{4}
$$

Eq. (6) can also be used to describe the upper portion of the PSD curve; i.e., particles coarser than $D_{2 n}$ will float in a matrix of fines if $D_{2 n}$ is coarser than $4 D_{n}$. Therefore, the voids between particles coarser than $D_{2 n}$ are filled by fine particles forming smaller voids. The truncated PSD determined in this fashion is then used to calculate the CSD as described in the "Constriction Size Distribution (CSD)" section.

\section{Particle Infiltration Depth}

A method will be described to estimate the distance a base soil particle of diameter $d$ can infiltrate into the 3D pore network model [Fig. 1(a)]. This particle infiltration depth is predicted by a probabilistic analysis of the particle sizes, from the base-soil PSD, and filter CSD. If a particle is smaller than a constriction, it can move through to the next pore. The probability that a base particle of diameter $d$ can pass a single, random constriction can be denoted by $p$, which is the cumulative probability of pore constrictions $>d$ (the fraction coarser than $d$ from the CSD). In the cubic pore model, if the particle does not move forward, there are four possible perpendicular exits (a sideways step). The probability of perpendicular (sideways) movement $P(1 S)$ is

$$
P(1 S)=\left[1-(1-p)^{4}\right](1-p)
$$

After this sideways movement, the particle again has a probability $p$ of moving forward in the direction of flow. Hence the conditional probability of a forward step, within the first pore or any of the neighboring four pores, $P(F \mid 1 S)$, is

$$
P(F \mid 1 S)=p+\left[1-(1-p)^{4}\right](1-p) p
$$

Eq. (8) can be extended to consider the probability of any number of sideways steps before a forward step, recognizing that there are now only three possible sideways exits from the pore. Continuing this process leads to (9), describing the overall probability of one forward step $P(F)$ in the direction of flow through the network model

$$
\begin{aligned}
& P(F)=p+\sum_{i=0}^{\infty}\left[1-(1-p)^{4}\right](1-p) p\left\{\left[1-(1-p)^{3}\right]\right. \\
& \cdot(1-p)\}^{i}
\end{aligned}
$$

where $i+1=$ number of perpendicular (sideways) steps before a forward step. Noting that the series term of (9) tends to zero as $i$ increases, it is possible to neglect higher-order terms beyond the first 3 or 4 terms. Silveira (1965) proposed a method to determine the number of confrontations with randomly generated pore constrictions, required to stop a particle moving forward through the filter, with a level of confidence $\bar{P}$. This equation can be adapted to consider the number of layers a particle can move through the pore network model $n$, based on the probability of passing one layer $P(F)$. Therefore

$$
n=\frac{\ln (1-\bar{P})}{\ln P(F)}
$$

The infiltration depth model requires a certainty level $\bar{P}$. Soria et al. (1993) have adopted a value of $98 \%$ in their analysis. Fig. 4 shows the probability of forward movement $P(F)$ from (9) against the probability of passing one constriction $p$. Also shown, on the right-hand $y$-axis, is the predicted number of layers $n$ a particle can infiltrate the filter [(10)] for various values of $\bar{P}$. The trend of the predicted infiltration depth is similar for each value of $\bar{P}$, tending to increase rapidly at some critical probability. Figs. 5 and 6 show that the CSD curve is more uniform than the PSD curve. Hence, the constriction diameter corresponding to the critical probability for various values of $\bar{P}$ should not vary greatly and the chosen value of certainty $\bar{P}$ would not be critical. A value of $\bar{P}=95 \%$ has been adopted in current modeling, as this gives a reasonable correlation with other models (Fig. 7). The predictions of Fig. 4 could be used to estimate the infiltration depth of particles into clean filters, based on the probability of a particle passing a single constriction $p$ and the predicted infiltration depth $n$.

The spacing of the void network is described as a "unit

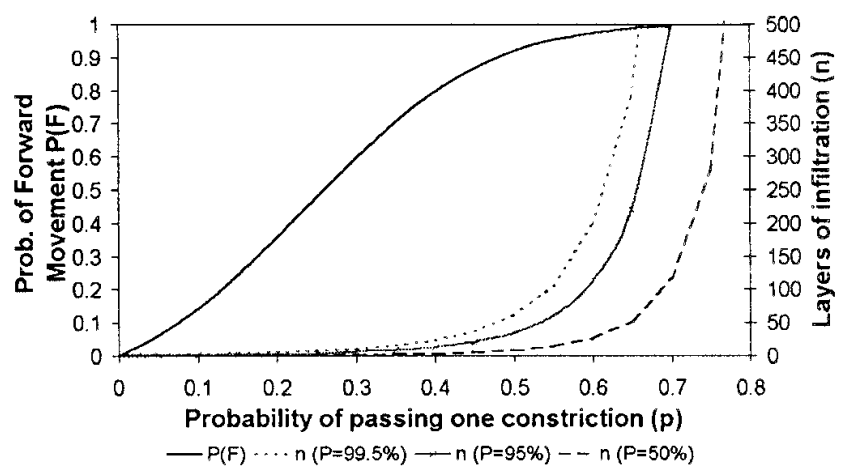

FIG. 4. Infiltration Model-Probability of Forward Movement and Predicted Depth of Infiltration

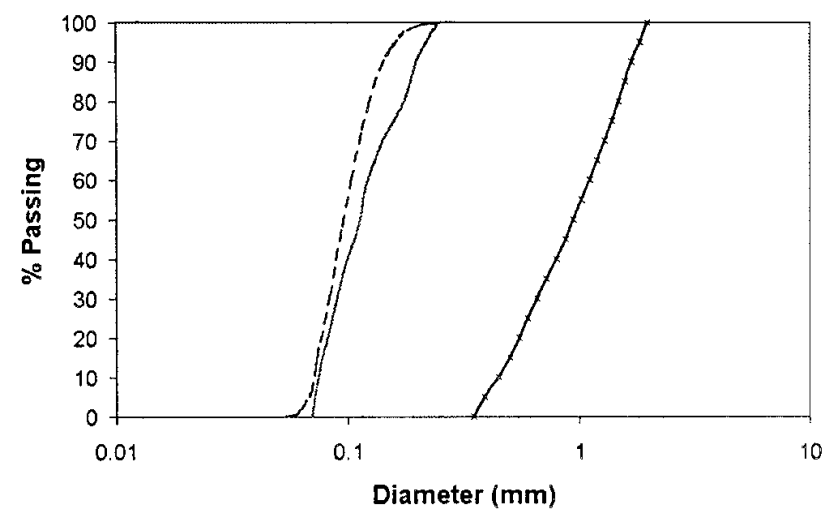

— PSD - Soria (1993) CSD - - New Model CSD

FIG. 5. Comparison of Calculated CSD with Measured CSD of Soria et al. (1993) 


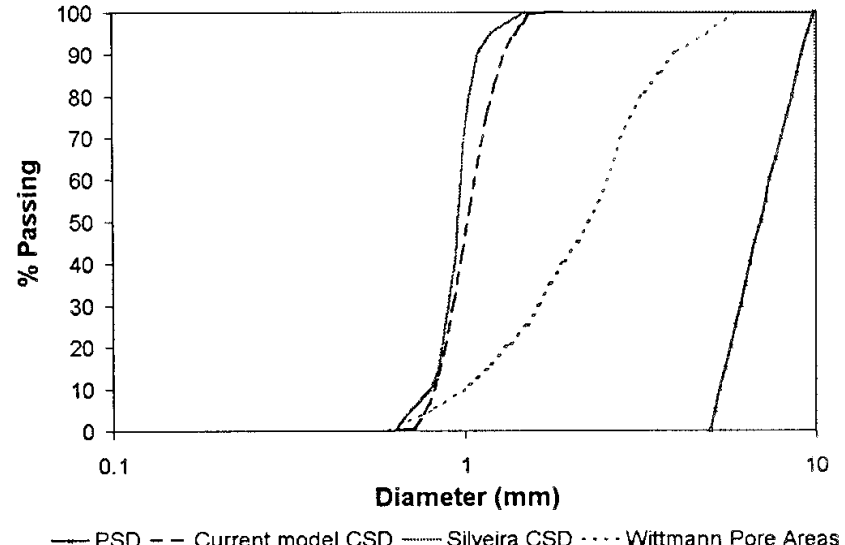

FIG. 6. Comparison of CSD Model with Measured Pore Sizes of Witmann (1979)

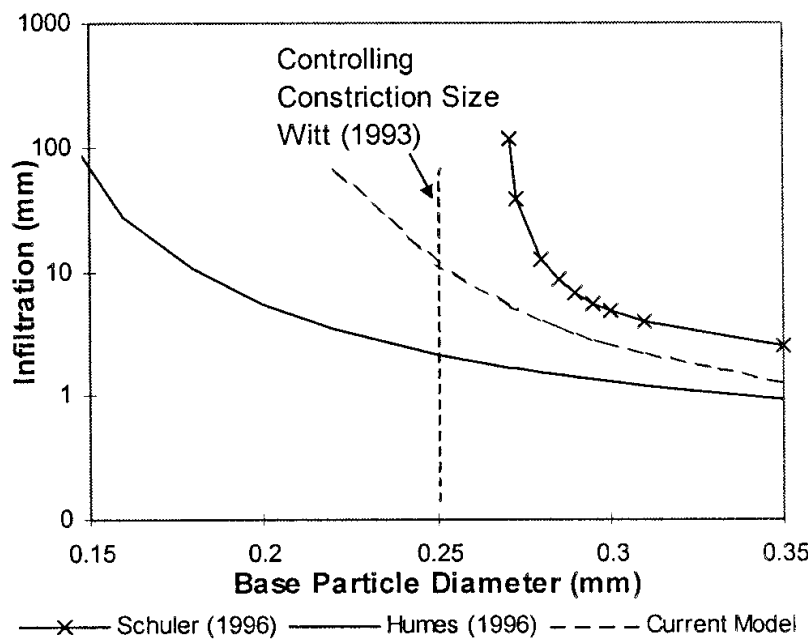

FIG. 7. Comparison of Base Particle Infiltration Models into WellGraded Filter, $C_{u}=6, D_{15}=1.3 \mathrm{~mm}$

step" in many previous models [Fig. 1(a)]. It is assumed that the distance between pores is constant, regardless of the pore size. Because constrictions form near the center of a filter particle and the next constriction will form near the center of the next particle, it seems reasonable to adopt the mean filter particle size by number $D_{f \text {, mean }}$ as the unit step. The expected length of infiltration $L$ of a base-soil particle into the filter is

$$
L=\frac{\ln (1-\bar{P})}{\ln P(F)} \cdot D_{f, \text { mean }}
$$

\section{Verification of Filter Void Model}

The proposed void model is compared with the published results of two experiments, which determined the constriction and void size distributions. The first is the data of Soria et al. (1993). These experiments used an indirect approach, passing uniform-sized particles through filters of varying length to find what particle size could pass different filtration lengths, then back-calculating the CSD. The PSD and measured CSD, along with the CSD calculated here, are shown in Fig. 5. The predicted CSD corresponds closely with that measured by Soria et al. (1993). The second experiment is that of Wittmann (1979), who filled a gravel with resin and cut slices through the solid material. Pore sizes on the revealed surface were measured using a planimeter. Because the slice is a plane through the filter material, the measured pore sizes include both the constrictions and the pores. Therefore, the calculated
CSD is expected to be more uniform, with sizes equal to the smaller measured pores, as shown in Fig. 6. The predicted CSD of the most-dense model of Silveira (1965) is also shown for comparison; the shape of the two CSD curves is similar, but the current model predicts slightly larger pores because the filter is not at its maximum density. In both cases, the relative density of the filter was estimated at $80 \%$.

The infiltration length model [(11)] can be compared to a number of other models for particle infiltration into a granular filter. The models of Schuler (1996) and Humes (1996) and the current model are able to predict the depth of infiltration of base particles of different diameters. Witt (1993) determined a controlling constriction size, which is a single constriction diameter that defines the smallest constriction that a particle is likely to encounter on any flow path. In other words, the controlling constriction size estimates a boundary between finer particles that will pass through the filter and coarser particles that are retained.

Most infiltration models give similar predictions for uniform materials $\left(C_{U}<3\right)$, but predictions become increasingly scattered for more broadly graded filters. The prediction of each of these models for the infiltration of particles into a wellgraded, gravelly sand, with a uniformity coefficient $C_{U}$ of 6 , is shown in Fig. 7. This graph describes the predicted depth of infiltration of particles of different diameters into the granular filter. As expected, all models predict that increasingly finer base-soil particles will infiltrate further into the filter. The model of Schuler (1996) tends to become asymptotic to the controlling constriction size of Witt (1993), thereby implying that no base particles finer than the controlling constriction size are retained. The model of Humes (1996) predicted that much finer particles are required for the same infiltration depth. As indicated in Fig. 7, the current model predicts infiltration depths similar to those of both Schuler (1996) and Humes (1996) for base particles exceeding the controlling constriction size of Witt (1993). For finer particles, the rapid increase of infiltration thickness (log scale) implies the potential risk of washout.

\section{PARTICLE TRANSPORT MODEL}

The maximum distance a particle of a certain diameter can infiltrate into the filter has been defined by (11). To describe the rate of movement of particles, a particle transport model is required, which must define the velocity and concentration of loose soil particles.

\section{Rate of Particle Transport}

Indraratna and Vafai (1997) developed a comprehensive particle transport model to simulate particle movement within granular media for noncohesive soils. Loose soil particles are assumed to be in suspension in a homogeneous slurry. The rate of movement of this slurry is controlled by governing differential equations of conservation of mass [(12)] and momentum [(13)]. The current model extends these concepts by incorporating the infiltration depth based on the cubic network structure, as explained earlier

$$
\begin{gathered}
\frac{d\left(\rho_{m} u\right)}{d z}=\frac{d \rho_{m}}{d t} \\
\sum F=\rho_{m} V_{m}\left(\frac{d u}{d t}+u \frac{d u}{d z}\right)
\end{gathered}
$$

In (12) and (13), $\rho_{m}, V_{m}$, and $u=$ slurry density, volume, and velocity, respectively; and $\sum F$ represents the external forces acting on the slurry, including the external hydraulic gradient and fluid drag. The Indraratna and Vafai (1997) model assumes that the pore water and loose particles move at the same speed 
and ignores the additional friction and energy loss due to particles in the flow. Because the slurry contains suspended particles, its viscosity $\eta$ is higher than that of clear water $\eta_{0}$. A relation developed by Happel and Brenner (1965) is adopted to determine the increase in viscosity due to interaction effects between the particles and pore walls, based on the slurry volumetric concentration $C$, as given by

$$
\frac{\eta}{\eta_{0}}=1+2.5 C\left(1+\frac{5 d D}{8(2 D-d)^{2}}\right)
$$

where $d$ may be taken as the mean particle diameter; and $D_{v}$ may be taken as the mean pore constriction diameter. This increase in viscosity causes an increased energy loss in the flow by decreasing the effective media permeability, hence

$$
k=k_{w} \frac{\eta_{0}}{\eta}
$$

where $k_{w}=$ media permeability to flow of pure water.

\section{Complete Transport Model}

By considering a number of elements at the base-filter interface, the movement of loose particles can be modeled by a finite-difference or finite-element analysis. The rate of particle erosion and movement is governed by (12) and (13). The geometric constraint to movement is modeled by the length of infiltration into the cubic network, incorporating (11). To use (11) to consider whether particles can move from one element to the next, it is necessary to define three base particle-size ranges:

- Fine particles that have an infiltration distance $L$ greater than the distance from the filter interface to the end of the element can pass through the element.

- Larger particles that have an infiltration distance smaller than above but can pass one unit step into the filter element [i.e., $n=1$ in (10)] are considered as moving into the next element and are then retained within the element. Once a particle is retained, it cannot be moved further. The coarsest particle within this range (corresponding to $n=1)$ is defined as the retained particle diameter $d_{r}$.

- Coarse particles that cannot pass one unit step into the filter remain in their current location.

As particles are captured within the filter, the diameter $d_{r}$ reduces and the filter is able to retain finer particles. This leads to a time-dependent description of the self-filtration process. To solve the governing differential equations [(12) and (13)], initial conditions are required for the flow velocity and slurry density. It is assumed that all particles finer than $d_{r}$ can erode and there is no selective erosion of finer particles. The maximum density of the slurry may be limited by the volume fraction of particles finer than $d_{r}$. However, very high flow concentrations have not been observed in laboratory testing. Calibration against laboratory observations suggests that the initial maximum slurry concentration be limited to $20-25 \%$ solids by volume. The initial flow velocity is determined by the Darcy equation, based on the applied head and base and filter permeabilities.

A finite-difference solution to the equations of motion will predict the changes in flow rate of the particulate slurry through the base soil and filter system. The volume of particles moving between elements is defined by the slurry density and velocity, and the size distribution of these loose particles is governed by a fraction of the base-soil PSD finer than diameter $d_{r}$. The base and filter particle-size distributions can be recalculated at each time step, based on the inflow and outflow slurry densities for each element (Indraratna and Vafai 1997)

$$
\begin{aligned}
& \left(V_{s-\text { out }}\right)_{j}^{t}=\left(V_{s-\text { out }}\right)^{t} \frac{P_{j}^{t-1}}{S_{r}} \\
& P_{j}^{t}=\frac{\left(V_{s}\right)_{j}^{t-1}-\left(V_{s-\text { out }}\right)_{j}^{t}}{V_{s}^{t}}
\end{aligned}
$$

In the above equations, $P_{j}=$ percentage of soil by total volume $V_{s}$ corresponding to a specific diameter $j ; S_{r}=$ percentage of particles finer than the retained diameter $d_{r}$; and $V_{s-\text { out }}=$ total volume of solid particles in the slurry leaving the element. The superscript denotes the time step in the finite-difference procedure. This analysis predicts the gradual change in particlesize distribution of the base and filter elements; hence, it describes what is occurring at the base-filter interface with time for the entire particle-size range.

Koenders and Williams (1992) presented (18) to determine the permeability of a granular soil, based on the mean particle diameter $D_{f, \text { mean }}$ and porosity $n_{e}$. This equation has been incorporated in the current particle-transport model, to determine the base-soil and filter permeability

$$
k=\frac{1}{\eta} D_{f, \text { mean }}^{2} n_{e} \chi\left(\frac{n_{e}}{1-n_{e}}\right)^{2}
$$

where $\chi=0.0035 \pm 0.0005$. The time-dependent change in porosity and relative density of each element can be estimated based on the original porosity and the volume of particles eroded from and retained in each element.

\section{APPLICATION OF MODEL}

To describe and test the validity of the model predictions, a comparison with a series of laboratory tests is described. In addition, the time-dependent predictions of the model are outlined, determining the time-dependent change in base and filter flow rate, mass transfer, permeability, and porosity. A method to apply the filtration model to a 2D flow net is described.

\section{Comparison with Laboratory Tests}

A large-scale filtration apparatus (500-mm diameter and $1,000-\mathrm{mm}$ high) was constructed to investigate the filtration of coarse and noncohesive materials typical of the filter and drainage zones within embankment dams. The tests described here employed a series of well-graded sands as the $150-\mathrm{mm}$ thick base soil and a well-graded gravel as the 800 -mm-thick filter. The filter was placed and compacted in 200-mm layers. A finer gravel was placed, approximately $5 \mathrm{~cm}$ deep, around the circumference of the filter interface to act as a "side material" to prevent the formation of preferential flow paths at the edge of the cylinder (Sherard et al. 1984). The base soil was placed and lightly compacted in a single layer of $150 \mathrm{~mm}$. A layer of geofabric was placed, and a small surcharge load of $2 \mathrm{kPa}$ was applied to the base soil to prevent heave. Erosion was induced by a uniform downward flow of $2.5 \mathrm{~L} / \mathrm{s}$. The flow was frequently interrupted (every minute) to avoid particle bridging and facilitate particle movement. After a 2-h test, the filter was sampled at increasing depths to determine the change in PSD due to infiltration of base-soil particles. The experiment was repeated for different base-filter combinations with varying filter retention ratios, $D_{15} / d_{85}$. In this paper, filter and base-soil particle sizes are denoted by $D$ and $d$, respectively.

The analytical model predicts the change in PSD of the base and filter soils during filtration. For effective filters, the PSD of the base and filter soils eventually reaches a stable distribution, where no further erosion occurs. The stable PSD of various filter elements, as predicted by the analytical model, can be compared with the measured PSD at the end of laboratory tests. Samples of filter material were carefully taken 
from the filtration apparatus at various depths from the filter interface to determine the laboratory PSD. The final predicted PSD of filter elements located at 5 and $20 \mathrm{~cm}$ from the basefilter interface is compared with the measured results for retention ratios $D_{15} / d_{85}$ of 4 and 7 , as shown in Figs. 8 and 9, respectively. The model predicts similar trends to those observed in the laboratory. As expected, the content of fines in the filter increases dramatically near the filter interface (at 5$\mathrm{cm}$ depth) as a self-filtration zone is formed. This long tail of fine particles indicates the retention of base-soil particles within the filter. At $20-\mathrm{cm}$ depth, there are fewer retained fines, indicating that the majority of particle retention occurs near the filter interface. In general, the model predicts a lesser amount of retained particles near the interface than measured; i.e., the laboratory curve at $5-\mathrm{cm}$ depth plots to the left of the model curve. This may be due to the bridging of soil particles over the filter voids or incomplete filtration. In addition, the assumption was made in the model that any particle with an infiltration distance $L[(11)]>1$ unit step will pass through to the next element. However, in reality, it is unlikely that all of these coarser particles will be located over a large pore constriction. In reality, small constrictions will retain some fine particles, which according to the probabilistic particle infiltration model, could move further.

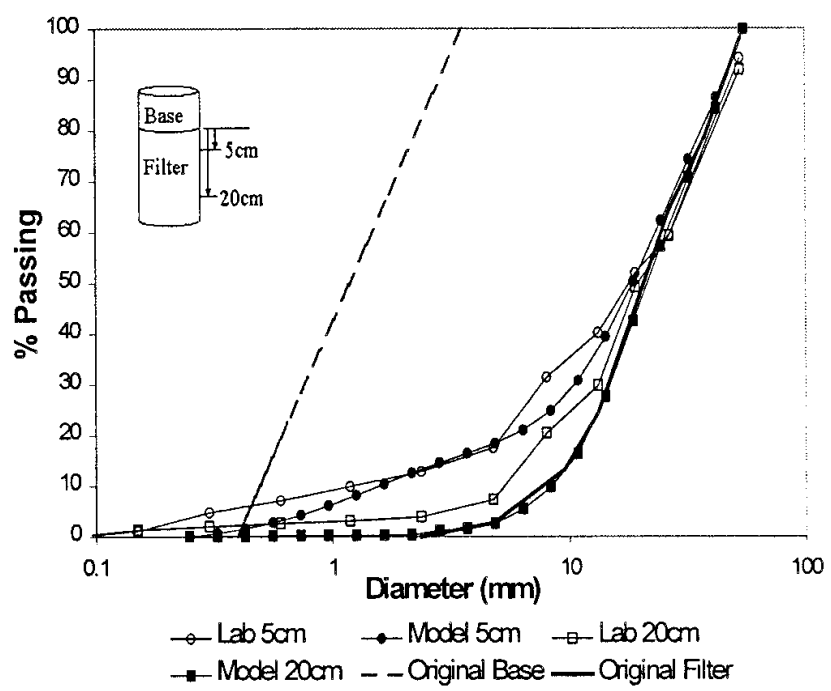

FIG. 8. Comparison of Predicted PSD and Laboratory Results$D_{15} / d_{85}=4$

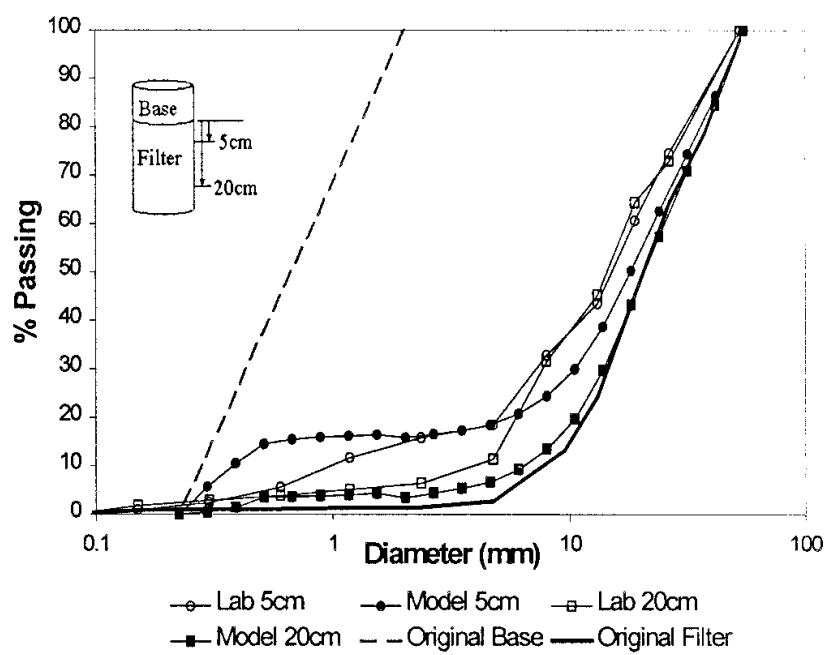

FIG. 9. Comparison of Predicted PSD and Laboratory Results$D_{15} / d_{85}=7$

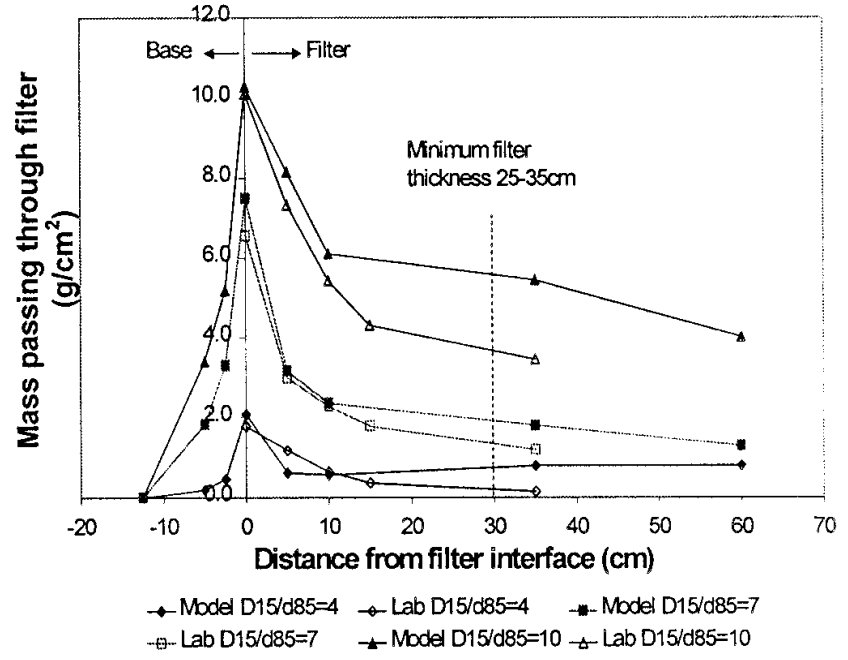

FIG. 10. Predicted and Measured Mass Loss during Filtration

Most important in the success or failure of filtration of broadly graded soils is the mass of base soil eroded before a self-filtration zone is formed. For the same experiment as outlined above, Fig. 10 shows both the predicted and the measured mass of base soil eroded and transported into and possibly through the filter, for three retention ratios $\left(D_{15} / d_{85}=4\right.$, 7 , and 10). The mass of base soil passing the filter, as plotted in Fig. 10, is defined as the total amount of mass passing a particular depth within the filter, from the base-filter interface. Hence, a sharp decrease in mass passing indicates particle retention whereas a constant mass passing suggests that the fine particles continue to move through the filter. The correlation between the model predictions and measured results is encouraging. The model overestimates the amount of mass passing entirely through the filter, for the reasons mentioned earlier. The model predicts that mass loss from the base soil occurs almost entirely within $5-10 \mathrm{~cm}$ of the filter interface. Within the filter, the eroded base soil is quickly retained in the self-filtering zone, which forms in the first 5-10 unit steps (or $15-30 \mathrm{~cm}$ ) of the filter. Each unit step of the filter void model is defined as the mean filter particle diameter $D_{f \text {, mean }}$ (usually in the range $D_{5}-D_{10}$ ). Beyond the self-filtering zone, the fine particles pass entirely through the filter. Therefore, the minimum filter thickness for effective filtration can be considered as 10 times the mean filter particle diameter $D_{f, \text { mean }}$.

\section{Model Prediction of Time-Dependent Filter Behavior}

An important feature of the model proposed in this paper is the ability to predict time-dependent changes in flow rate, permeability, and porosity of the base and filter soils. The model predictions of the laboratory experiments, described in the "Comparison with Laboratory Tests" section, are discussed here. The analysis uses the same filter material as described above $\left(D_{15}=10.1 \mathrm{~mm}\right.$ and $\left.C_{u}=2.5\right)$, with four different base soils, graded such that $C_{u}=3$, and $d_{85}$ is selected to obtain filtration ratios of $D_{15} / d_{85}=4,7,10$, and 12 . The initial porosity of all the base soils was assumed constant at $35 \%$ for ease of comparison, and the initial filter porosity was estimated at $40 \%$. The hydraulic gradient in each simulation was varied to ensure an initial flow rate of $0.2 \mathrm{~L} / \mathrm{s} / \mathrm{m}^{2}$ for each base-filter combination.

The analytical model predicts the time-dependent erosion and retention within the base-soil-filter system. The final predicted base-soil erosion and mass capture within the filter has been described in Fig. 10 for effective filter retention ratios $\left(D_{15} / d_{85}=4,7\right.$, and 10). Fig. 11 shows the time-dependent mass of base soil passing out of the second filter element (10 


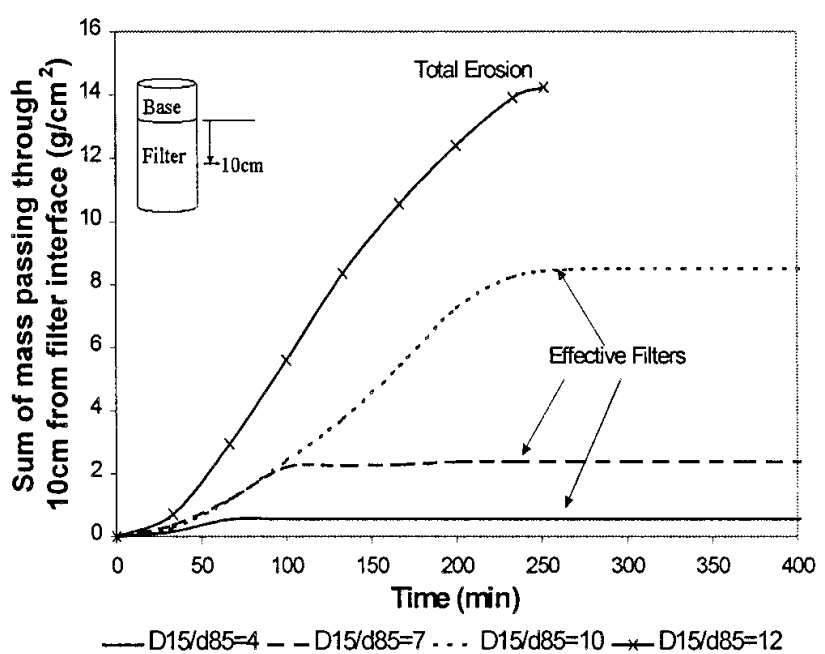

FIG. 11. Mass Passing $10 \mathrm{~cm}$ from Filter Interface with Time

$\mathrm{cm}$ from the filter interface). This depth was chosen to exclude the majority of mass retained within the self-filtration zone. For a low retention ratio $\left(D_{15} / d_{85}=4\right)$, the self-filtration zone stabilizes quickly and little mass passes through the filter. For increasingly finer base soils, the time taken for the self-filtration zone to prevent particle movement and the quantity of particles passing into the filter increases greatly. The finest base soil $\left(D_{15} / d_{85}=12\right)$ is too fine to be retained by the filter; hence, the filter is ineffective. This material exhibits continuous erosion, and the cumulative mass passing through the filter increases until complete erosion.

Fig. 12 shows the predicted change in flow rate through the four base-soil and filter combinations. The coarsest base soil $\left(D_{15} / d_{85}=4\right)$ initially exhibits an increase in flow rate as the fines of the base soil wash out through the filter. Following this initial increase, the filter permeability decreases as basesoil particles are retained and the flow rate diminishes again. The flow rate stabilizes at a value only slightly greater than the initial flow rate. A finer base soil $\left(D_{15} / d_{85}=7\right)$ shows unstable flow for a longer period and a greater increase in flow. As the filter stabilizes, the flow rate becomes constant at a significantly higher value than that of the $D_{15} / d_{85}=4$ case, which is due to a greater loss of base soil, therefore resulting in a greater increase in the base-soil permeability. The base soil with $D_{15} / d_{85}=10$ does eventually stabilize but indicates a fivefold increase in flow rate and a large loss of mass. Although eventually effective, this is an example of a borderline filter. The finest base soil $\left(D_{15} / d_{85}=12\right)$ is not retained by the filter. The flow rate increases continually throughout the simulation, indicating that particle erosion will accelerate until complete washout as the porosity of the base soil increases.

Fig. 13 shows the predicted, time-dependent change in permeability and porosity of the base soil during filtration. In all three simulations where a stable interface results $\left(D_{15} / d_{85}=4\right.$, 7 , and 10), the base-soil permeability increases, attaining a constant value as the filter interface stabilizes. The permeability of the coarsest base soil $\left(D_{15} / d_{85}=4\right)$ increases from 0.075 to $0.46 \mathrm{~mm} / \mathrm{s}$ during filtration. The permeability of the borderline base soil $\left(D_{15} / d_{85}=10\right)$ increases from 0.02 to 0.25 $\mathrm{mm} / \mathrm{s}$, and this increase is almost $100 \%$ more than the coarsest base soil. The permeability of the unsuccessful base soil $\left(D_{15} / d_{85}=12\right)$ increases steadily until failure, indicating continued erosion and limited particle capture in the filter to resist erosion. The time-dependent change in base-soil porosity is also shown in Fig. 13. For the effective base-soil-filter combinations, the base-soil porosity initially increases steadily as mass is lost, approaching a constant value as the self-filtration zone is formed. The coarsest base soil $\left(D_{15} / d_{85}=4\right)$ shows the greatest resistance to erosion and is characterized by the lowest increase in porosity with time. As expected, the rate of erosion (indicated by the change in porosity) increases as the size of base soil decreases, in relation to the same filter material. In the case of unsuccessful filtration, the porosity increases stead-

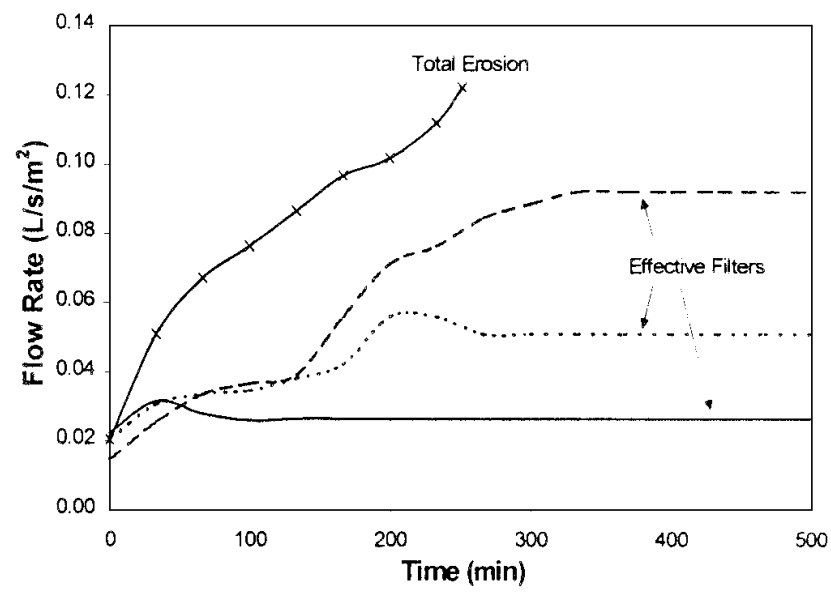

D $15 / \mathrm{d} 85=4 \ldots \mathrm{D} 15 / \mathrm{d} 85=7-\mathrm{D} 15 / \mathrm{d} 85=10 \rightarrow \mathrm{D} 15 / \mathrm{d} 85=12$

FIG. 12. Predicted Change in Flow Rate with Time

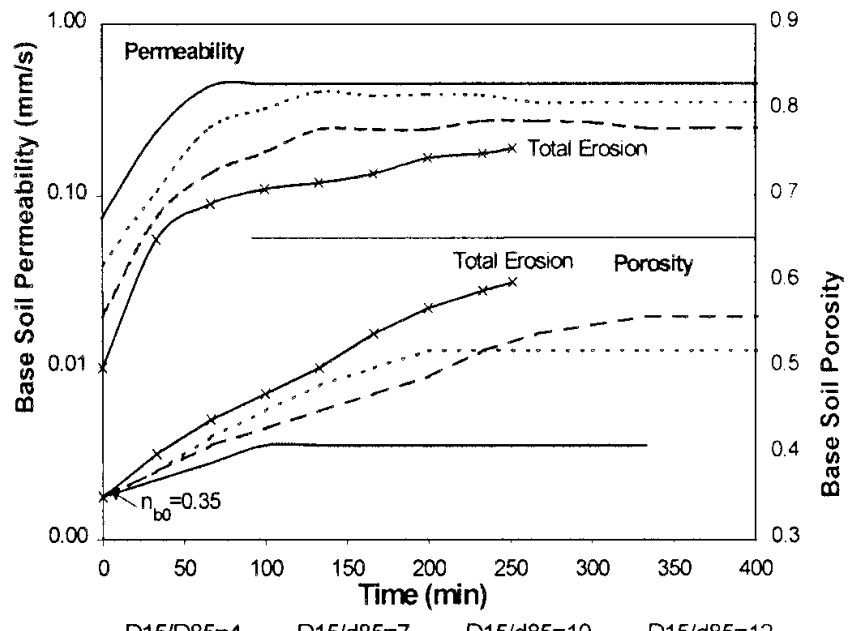

FIG. 13. Change in Permeability and Porosity of Base Soil with Time

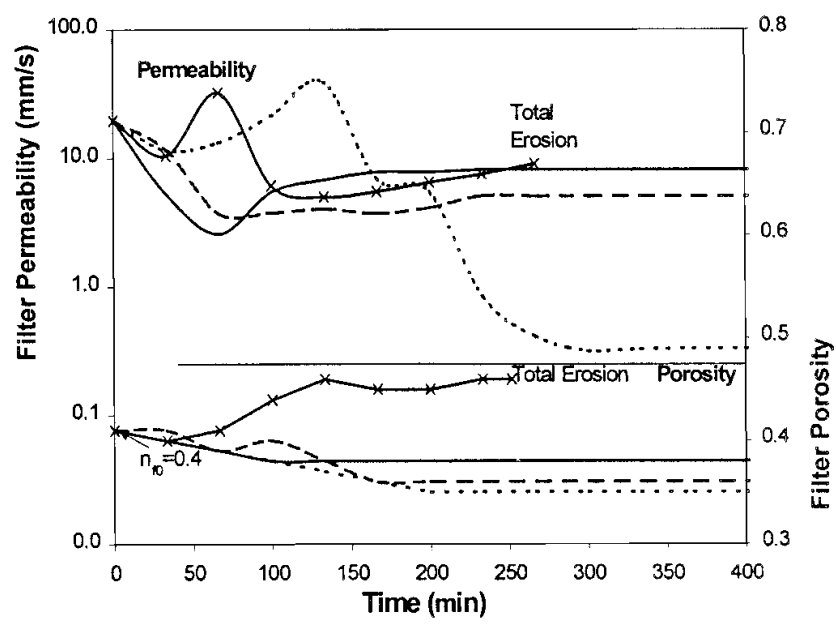

-D15/d85=4 - D15/d85=7 . . D15/d85=10 *D15/d85=12

FIG. 14. Change in Permeability and Porosity of Filter with Time 
ily until a value of 0.6 is reached (i.e., the definition of failure in the model).

The permeability of the filter interface (Fig. 14) initially decreases as some base-soil particles are captured. However, the filter material is internally unstable, containing approximately $5 \%$ loose fines. The rate of wash out of these filter fines becomes larger than the mass rate of base soil retained by the filter; hence, the filter permeability increases again. In successful filter combinations, after this initial fluctuation, the loose filter fines have almost completely been washed out of the filter element and the permeability again decreases as more base-soil particles are captured. The borderline base soil $\left(D_{15} / d_{85}=10\right)$ leads to a significantly lower final filter permeability than the two coarser base soils, because a larger mass of finer particles is eventually captured in the filter. The permeability of the filter element for the unsuccessful combination $\left(D_{15} / d_{85}=12\right)$ exhibits similar trends initially to the successful filters as base-soil particles within the filter reduce the permeability. However, failure is predicted before the interface can stabilize.

The time-dependent porosity of the filter at the interface (Fig. 14) initially decreases as base-soil particles migrate into the filter. For the three effective filtration cases, $D_{15} / d_{85}=4$, 7 , and 10 , the filter porosity stabilizes at a value lower than the initial as the retained mass prevents further erosion. The reduction in the filter porosity indicates the amount of base soil retained within the filter. The filter porosity of the unsuccessful case increases to a value greater than the initial porosity of $40 \%$. This is because, as previously mentioned, the filter material is internally unstable. This leads to an increase in the filter porosity as the filter fines are washed out.

The model predictions show that, irrespective of the initial retention rates of base soil in the filter, an effective filter is characterized by a stable (constant) permeability and porosity with time. This prediction describes what occurs within a granular filter when water first starts flowing through the base soil (e.g., first filling of a dam reservoir). If a further perturbation occurs (such as cracking of the core, changes in seepage patterns, and change in the reservoir water chemistry), a subsequent load of base soil may reach the filter. The model could then be used to consider the effect of this new flow of basesoil particles into the filter material that has already been modified by previous infiltration. If a filter is predicted to be effective in the first instance, the model may predict a further decrease in filter porosity and permeability until steady-state conditions are reached once again. In this way, the full life of the filter can be simulated. The base soils and filter described here are coarse, noncohesive sands and gravels. Similar trends in experimental results and model predictions have been obtained for several finer, noncohesive materials.

\section{Practical Applications}

The model described in this paper predicts the time-dependent transport and capture of noncohesive base soils, within granular filters. Analytical models can have an important application in the design and construction of granular filters for large projects such as embankment dams. Modeling predictions can give a designer a detailed picture of what may occur during the design life of the structure, rather than relying entirely on empirical grain-size criteria. Model predictions can be compared with the actual behavior to verify that the filter is performing as expected. The model developed in this paper describes filtration of noncohesive base soils. The rate of particle capture could be modified to consider progressive accumulation of fine, cohesive particles by physicochemical effects (Reddi and Bonala 1997).

The model can be applied to describe filtration within a geotechnical structure. A seepage analysis will define the ini-

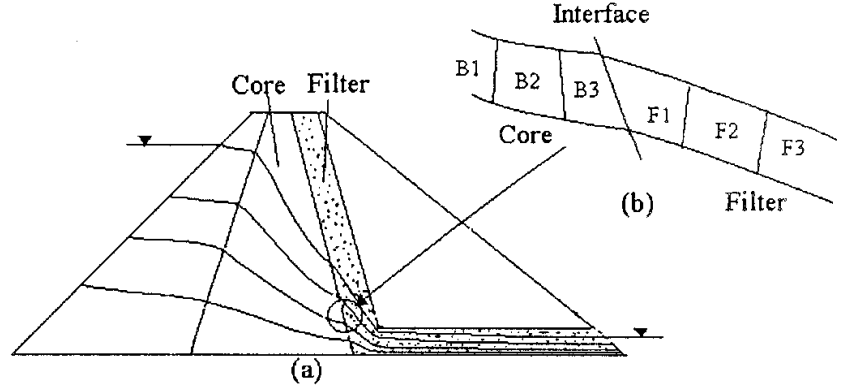

FIG. 15. (a) Seepage through Embankment Dam; (b) Discrete Elements along Flow Path [after Vafai (1996)]

tial flow paths, flow rates, and hydraulic gradients expected within the structure [Fig. 15(a)]. Flow occurs only along flow paths; hence, it is possible to apply the filtration model to this pseudo-1D flow. A number of elements can be considered at the base-soil-filter interface for each flow path [Fig. 15(b)], and the time-dependent changes within these materials examined. After a reasonable period, the seepage analysis can be repeated to determine the changes to the flow paths and hydraulic gradients within the structure and the new parameters updated in the filtration model. The model has been successfully used to examine the behavior of filter zones in embankment dams and predict the internal stability of broadly graded granular materials.

\section{CONCLUSIONS}

A revised analytical model for the movement of noncohesive base-soil particles into granular filters, extending the particle transport concept of Indraratna and Vafai (1997), has been described. The model consists of three important sections:

- A filter void model based on a 3D cubic network proposed by Schuler (1996) — the size of pore constrictions linking the pores is determined from the filter particle-size distribution (by surface area) and relative density.

- A deterministic equation for particle infiltration into the filter model based on the probability of movement of a particle through the filter void constrictions - this equation defines the retention of particles within the filter.

- Particle transport equations of conservation of mass and momentum, proposed earlier by Indraratna and Vafai (1997), which have been modified to consider the increased flow viscosity due to suspended particles in the pore water-the particle transport model defines the erosion and transport of base-soil particles.

Within broadly graded soils, some fine particles may not contribute to the skeleton of fixed particles, which form the structure of the soil, but are loose within the pores of this skeleton and do not contribute to particle capture during filtration. These particles should not be considered when determining the size of constrictions in the filter voids. At the other extreme, some coarse particles may be enmeshed in a matrix of fines. In this case, the fines fill the voids formed by these coarse particles. A method has been described to determine which particle sizes should be excluded from the PSD when determining the CSD of a broadly graded material.

The model predicts the time-dependent rate of erosion of base-soil particles and retention within a granular filter and predicts, for a suitable base-soil-filter combination, the formation of a self-filtering layer at the filter interface. As basesoil particles are captured within the filter, void sizes will decrease and finer base-soil particles will be retained. This process can only be modeled by a time-dependent analysis. This leads to the formation of a stable, self-filtering layer ap- 
proximately 5-10 "unit steps" (or $15-30 \mathrm{~cm}$ ) in thickness, where each unit step corresponds to the mean filter particle diameter by number $D_{f \text {,mean }}$ (usually between $D_{5}-D_{10}$ ). Beyond this self-filtering layer, there is limited retention of particles, suggesting that increasing the filter thickness does not greatly affect the capture of particles. Time-dependent particle capture and changes in flow rate, permeability, and porosity are calculated by the model, leading to a full description of the filtration process.

Comparison with laboratory results and previously published experimental findings has shown that the current model predicts particle movement and capture similar to the measured data for noncohesive, uniform, and well-graded base and filter materials. The model is able to predict particle movement and changes in flow rate, permeability, and porosity for noncohesive, uniform, and broadly graded base and filter materials and is a significant extension of the original Indraratna and Vafai (1997) analytical method. In addition, this revised model has been shown to predict internal stability to some extent, based on fine filter particle washout.

\section{ACKNOWLEDGMENT}

The writers wish to thank the Snowy Mountains Engineering Corp. for its commitment to advancing knowledge in dam design through financial support of this Australian Research Council-SPIRT research program.

\section{REFERENCES}

Delgado Ramos, F. (2000). "Laboratory simulation of the internal erosion phenomenon to determine the variables that influence the efficiency of filters for cohesive soils." $\mathrm{PhD}$ thesis, University of Granada, Granada, Spain (in Spanish).

Giroud, J. (1996). "Granular filters and geotextile filters." Proc., Geofilters '96, Comptes Rendus, J. Lafleur and A. Rollin, eds., Bitech Publications, Montreal, Canada, 565-680.

Happel, J., and Brenner, H. (1965). Low Reynolds number hydrodynamics, Noordhoff, Leyden, The Netherlands.

Humes, C. (1996). "A new approach to compute the void-size distribution curves of protective filters." Proc., Geofilters '96, Comptes Rendus, Lafleur and Rollin, eds., Bitech Publications, Canada, 57-66.

Indraratna, B., and Locke, M. (2000). "Analytical modelling and experimental verification of granular filter behaviour." Filters and drainage in geotechnical and geoenvironmental engineering, W. Wolski and J. Mlynarek, eds., Balkema, Rotterdam, The Netherlands, 3-26.

Indraratna, B., and Vafai, F. (1997). "Analytical model for particle migration within base soil-Filter system." J. Geotech. and Geoenvir. Engrg., ASCE, 123(2), 100-109.

Koenders, M., and Williams, A. (1992). "Flow equations for particle fluid mixtures." Acta Mechanica, 92(1), 91-116.

Reddi, L. (1997). "Particle transport in soils: Review of significant processes in infrastructure systems." J. Infrastruct. Sys., ASCE, 3(2), 7886.

Reddi, L., and Bonala, M. (1997). "Analytical solution for fine particle accumulation in soil filters." J. Geotech. and Geoenvir. Engrg., ASCE, 123(12), 1143-1152.

Schuler, U. (1996). "Scattering of the composition of soils. An aspect for the stability of granular filters." Proc., Geofilters '96, Comptes Rendus, Lafleur and Rollin, eds., Bitech Publications, Canada, 21-34.

Sherard, J., Dunnigan, L., and Talbot, J. (1984). "Basic properties of sand and gravel filters." J. Geotech. Engrg., ASCE, 110(6), 684-700.

Silveira, A. (1965). "An analysis of the problem of washing through in protective filters." Proc., 6th Int. Conf. Soil Mech. and Found. Engrg., ICSMFE, Montreal, Canada, Vol. 2, 551-555.

Silveira, A., de Lorena Peixoto, T., and Nogueira, J. (1975). "On void size distribution of granular materials." Proc., 5th Pan-American Conf.
Soil Mech. and Found. Engrg., PASMFE, Buenos Aires, Argentina, $161-176$.

Skempton, A., and Brogan, J. (1994). "Experiments on piping in sandy gravels." Géotechnique, London, 44(3), 449-460.

Soria, M., Aramaki, R., and Viviani, E. (1993). "Experimental determination of void size curves." Filters in geotechnical and hydraulic engineering, J. Brauns, M. Heibaum, and U. Schuler, eds., Balkema, Rotterdam, The Netherlands, 43-48.

Terzaghi, K. (1922). "Der Grundbruch an Stauwerken und Seine." Verhuntung Forcheimer-Nummer Wasserkr, 17, 445-449 (in German).

Vafai, F. (1996). "Analytical modelling and laboratory studies of particle transport in porous media." $\mathrm{PhD}$ thesis, University of Wollongong, Wollongong, Australia.

Witt, K. (1993). "Reliability study of granular filters." Filters in geotechnical and hydraulic engineering, J. Brauns, M. Heibaum, and U. Schuler, eds., Balkema, Rotterdam, The Netherlands, 35-42.

Wittmann, L. (1979). "The process of soil filtration-Its physics and the approach in engineering practice." Proc., 7th Eur. Conf. Soil Mech. and Found. Engrg., BGS, London, 303-310.

\section{NOTATION}

The following symbols are used in this paper:

$C=$ volume concentration of slurry flow;

$D_{V L D}=$ constriction diameter from least-dense packing model of four particles;

$D_{V M D}=$ constriction diameter from most-dense packing model of three particles;

$D_{f, \text { mean }}=$ mean filter particle diameter (calculated from PSD by number rather than mass);

$D_{i}=$ diameter of $i \%$ coarsest filter particle (e.g., $D_{15}$ );

$D_{\max }=$ largest particle diameter;

$D_{\mathrm{v}}=$ void constriction diameter;

$d_{i}=$ diameter of $i \%$ coarsest base-soil particle (e.g., $d_{85}$ );

$d_{r}=$ maximum particle diameter entering element and then retained;

$k=$ permeability;

$L=$ length particle can infiltrate $3 \mathrm{D}$ pore model;

$n=$ number of layers (or forward steps) particle will infiltrate 3D pore model;

$n_{e}=$ porosity;

$\bar{P}=$ certainty in probabilistic infiltration depth model;

$P(F)=$ probability of forward step (movement of particle from one void to another in direction of flow) in 3D pore model;

$P(F \mid 1 S)=$ probability of forward step from either initial void or after one perpendicular (sideways) step, in 3D pore model;

$P_{i}=$ probability of occurrence (frequency) of particles of diameter $i$ (from PSD);

$P_{\mathrm{v}}=$ probability of occurrence (frequency) of void constrictions of diameter $D_{\mathrm{v}}$;

$p=$ probability that particle of diameter $d$ can pass through random pore constriction;

$R_{D}=$ relative density;

$r_{i}=$ number of times particles of diameter $D_{i}$ appear in combination of particles forming constriction;

$S_{n}=$ mass fraction passing diameter $D_{n}$ from PSD;

$u=$ slurry velocity;

$V_{m}=$ volume of slurry within element;

$\left(V_{s}\right)_{j}=$ volume of solid particles in slurry in element $j$;

$\left(V_{s-\text { out }}\right)_{j}=$ volume of solid particles in slurry leaving element $j$;

$\eta=$ viscosity;

$\rho_{m}=$ slurry density;

$\Sigma F=$ sum of forces on seepage flow within element; and $\chi=$ nondimensional permeability coefficient. 